\title{
Extending the Interval of Natalizumab Dosing: Is Efficacy Preserved?
}

\author{
Marinella Clerico ${ }^{1}$ - Stefania Federica De Mercanti ${ }^{1}$ - Alessio Signori ${ }^{2} \cdot$ Marco ludicello $^{1} \cdot$ Cinzia Cordioli $^{3}$. \\ Elisabetta Signoriello ${ }^{4}$. Giacomo Lus ${ }^{4}$. Simona Bonavita ${ }^{5}$. Luigi Lavorgna ${ }^{5}$. Giorgia Teresa Maniscalco ${ }^{6}$. \\ Erica Curti ${ }^{7}$. Lorena Lorefice ${ }^{8}$. Eleonora Cocco $^{8}$ - Viviana Nociti ${ }^{9} \cdot$ Massimiliano Mirabella ${ }^{9} \cdot$ Damiano Baroncini $^{10}$. $^{2}$ \\ Giorgia Mataluni ${ }^{11}$ - Doriana Landi ${ }^{11} \cdot$ Martina Petruzzo $^{12} \cdot$ Roberta Lanzillo $^{12} \cdot$ Ilaria Gandoglia ${ }^{13} \cdot$ Alice Laroni $^{14}$. \\ Rita Frangiamore ${ }^{15}$. Arianna Sartori ${ }^{16} \cdot$ Paola Cavalla $^{17} \cdot$ Gianfranco Costantini $^{17} \cdot$ Maria Pia Sormani $^{2}$. \\ Ruggero Capra ${ }^{3}$
}

Published online: 26 August 2019

(C) The American Society for Experimental NeuroTherapeutics, Inc. 2019

\begin{abstract}
Extending the natalizumab interval after the 24th administration could reduce the risk of progressive multifocal leukoencephalopathy (PML). The objective is to evaluate the noninferiority of the efficacy of an extended interval dosing (EID) compared with the standard interval dosing (SID) of natalizumab. It is an observational, multicenter (14 Italian centers), retrospective cohort study, starting from the 24th natalizumab infusion to the loss of follow-up or 2 years after baseline. Patients were grouped in 2 categories according to the mean number of weeks between doses: $<5$ weeks, SID; $\geq 5$ weeks, EID. Three hundred and sixty patients were enrolled. Median dose interval (MDI) following 24th infusion was 4.7 weeks, with a bimodal distribution (modes at 4 and 6 weeks). Two hundred and sixteen patients were in the SID group (MDI = 4.3 weeks) and 144 in the EID group (MDI 6.2 weeks). Annualized relapse rate was 0.060 (95\% CI $=0.033-0.087)$ in the SID group and $0.039(95 \% \mathrm{CI}=$ $0.017-0.063$ ) in the EID group. The non-inferiority of EID versus SID was satisfied. In conclusion, there is no evidence of a reduced efficacy of natalizumab in an EID setting. This observation confirms previous results and together with the emerging evidence of a reduced risk of PML associated to an EID, supports the need of a randomized study to assess the need to change the standard of the natalizumab dosing schedule.
\end{abstract}

Key Words Multiple sclerosis $\cdot$ natalizumab $\cdot$ extended dose $\cdot$ progressive multifocal leukoencephalopathy $\cdot$ efficacy

\section{Introduction}

Natalizumab (Tysabri; Biogen-Idec, Cambridge, MA, USA) is a humanized monoclonal antibody directed against the $\alpha 4$ subunit of $\alpha 4 \beta 1$ and $\alpha 4 \beta 7$ integrins, localized on the surface of circulating mononuclear cells, inhibiting the binding to

Stefania Federica De Mercanti and Alessio Signori share cosecond authorship. Ruggero Capra and Maria Pia Sormani share cosenior authorship.

Electronic supplementary material The online version of this article (https://doi.org/10.1007/s13311-019-00776-7) contains supplementary material, which is available to authorized users.

Stefania Federica De Mercanti

sdemercanti@yahoo.it

Extended author information available on the last page of the article their endothelial receptors, thus limiting the entry of lymphocytes through the blood-brain barrier (BBB) [1]. This leads to the reduction of the central nervous system (CNS) inflammation in the treatment of relapsing forms of multiple sclerosis (MS), with reduction of clinical and radiologic disease activities [2,3]. Nevertheless, this mechanism of action impacts on the CNS immunosurveillance [4], which could be responsible for the development of progressive multifocal leukoencephalopathy (PML), a rare and potentially lethal infection caused by the John Cunningham (JC) virus $[5,6]$. PML risk is higher in JCV-positive patients (index $>1.5$ ), after 24 doses, and in those who previously received immunosuppressive drugs [7].

Pharmacological analysis [8] revealed a nonlinear pharmacokinetic of natalizumab, resulting in a recommended fixed dose of $300 \mathrm{mg}$ administered intravenously every 4 weeks, ensuring continuous maximal $\alpha 4 \beta 1$ integrin receptor saturation. Following a single natalizumab administration, serum 
concentrations rise rapidly followed by a long-standing final phase during which serum concentrations reduce to values of about $3 \mu \mathrm{g} / \mathrm{mL}$ over 4 weeks [8]. A complete receptor desaturation, defined as saturation lower than $50 \%$, occurs more than 8 weeks after drug administration when natalizumab serum concentrations are below $1 \mu \mathrm{g} / \mathrm{mL}$ [9], whereas detectable natalizumab levels are seen on average at 8 weeks and are supposed to be even higher in patients who received repeated administration, in consideration of the rising concentration over time [2, 10-12]. A recent prospective observational cohort study [13] showed that the majority of natalizumab-treated patients showed high $(>10 \mu \mathrm{g} / \mathrm{mL})$ drug serum concentrations at time of redosing.

MS reactivation or a rebound effect has been reported within 6 months after natalizumab discontinuation [14], with a peak at around 10 to 12 weeks following drug withdrawal [15], especially in patients with high disease activity the year before treatment starts [14]. This timing could indicate that natalizumab receptor saturation is more linked to natalizumab mechanism of action than to serum concentration [12, 16, 17].

The European Medical Agency (EMA) stated that after the 24th natalizumab dose, patients should be informed again about the PML and MS reactivation/rebound risk related to natalizumab continuation or withdrawal, and they are asked to provide a standardized written consent form to continue this therapy [18-20].

Different strategies were proposed to minimize the risk of both PML and rebound in JCV-positive MS patients who decided to continue natalizumab treatment after 24 doses. Among these proposals, it was suggested to extend the interval between natalizumab administrations. Recently neurologists started to prescribe different extended interval dosing (EID) programs, without a precise coding. Two retrospective analysis $[15,21]$ on data from a multicenter cohort of MS natalizumab-treated patients with different EID schedules pointed out that dosing intervals ranging from 6 to 8 weeks do not reduce efficacy of natalizumab, but data on PML risk mitigation are not conclusive.

An unmet need is to define a strategy to maintain the high efficacy of natalizumab, reducing the risks of PML and clinical reactivation. The objective of this study is to evaluate the noninferiority in controlling disease activity of an EID of natalizumab in a large cohort of MS patients treated with natalizumab for 24 weeks.

\section{Methods}

Ethical committees of each participating hospital or university approved the study protocol. The study was registered with the Comitato Etico Interaziendale A.O.U. San Luigi Gonzaga, Orbassano, Italy (No. 39/2016). All participating patients provided written informed consent.
This study is a spontaneous, observational, multicenter cohort study with a retrospective analysis of information collected in standard clinical practice. A total of 360 adult patients with clinically definite relapsing MS (R-MS), according to revised McDonald's criteria [22], who received at least 24 natalizumab doses and decided to continue natalizumab, were recruited from 14 Italian MS centers between March 2007 and March 2018. All patients within 1 month after the 24th natalizumab administration underwent brain MRI to exclude PML signs and to detect MS brain activity.

Exclusion criteria were pregnancy, severe depression, and alcohol or drug addiction. Data for each patient were collected by the evaluating neurologist in an electronic case report form (CRF) located on the server of the coordinating center (Department of Clinical and Biological Sciences, University of Turin).

The day of the 24th natalizumab dose was considered the study baseline. Patients were examined after 24th natalizumab administration and according to good clinical practice thereafter. Assessments included a physical and a neurologic examination with the Expanded Disability Status Scale (EDSS) evaluation [23]. Safety assessments, performed at each visit, included vital signs, concomitant medication use, and collection of information on adverse effect. Patients also underwent hematologic and biochemical tests every month. In case of exacerbations or adverse effects during follow-up, patients were asked to contact the MS center; MS exacerbations were treated with high-dose intravenous methylprednisolone sodium succinate if needed. Exacerbation was defined as the occurrence of a new neurologic symptom or a worsening of an old one, with an objective change of at least 1 point on the EDSS, which lasted at least for $24 \mathrm{~h}$ in the absence of fever and followed a period of clinical stability or improvement of at least 30 days.

Two treatment strategies were compared: the standard interval dose (SID) versus an extended interval dose (EID). Patients were assigned to 1 of the 2 treatment arms according to the mean number of weeks between doses received during the first 6 months after baseline. Patients were assigned to the SID arm if the mean interval between doses was $<5$ weeks, whereas they were assigned to the EID arm if the mean interval between doses was $\geq 5$ weeks. The 5 -week cutoff was defined a priori since it is the midpoint between the theoretical SID ( 4 weeks) and the theoretical EID ( 6 weeks). The followup period started at the 24-month infusion and ended at loss to follow-up or 2 years after baseline, whichever occurs first.

The primary outcome of the study was the number of clinical relapses and summarized as an annualized relapse rate (ARR). ARR is defined as the total number of relapses count on person/years. 


\section{Statistical Analyses}

Two causal contrasts were assessed: the intention-to-treat (ITT) effect of EID versus SID was evaluated comparing the 2 groups according the definition of SID and EID obtained in the first 6 months after baseline. Also, a per-protocol (PP) analysis was run, in which the dose intervals between baseline and the end of follow-up ( 2 years) or the first relapse, whichever occurred first, were used to estimate the mean intervals between doses. The reason to stop the follow-up to the first relapse to estimate the dose intervals is to reduce the potential bias deriving from a possible change in the dosing strategy following a relapse. This study was underpowered to detect noninferiority, since thousands of patients would be required for patients with such a low relapse rate, as those in natalizumab therapy. We anyway defined as satisfied a noninferiority of EID versus SID if the upper limit of the $95 \%$ CI of the ARR in the EID group did not exceed the mean ARR of the SID group by 0.02 relapse/year.

Baseline characteristics were compared between groups by a Mann-Whitney $U$ test. ARR during follow-up was estimated and compared between groups by a multivariate Poisson regression model. Time to first relapse was compared between groups by a Cox multivariate model.

Sensitivity analyses were run i) excluding patients in the gray zone of a mean interval dosing around 5 weeks (3238 days), ii) considering the mean dose interval as a continuous variable, and iii) using propensity score (PS) as covariate in the regression models to adjust for between-groups differences at baseline. Propensity score was built using logistic regression model with dose group indicator as dependent variable and the baseline characteristics as independent variables. Stata (v.14; StataCorp) was used for the computation.

\section{Results}

In total, 360 patients with a median follow-up of 2.2 years (range 0.03-8.8) were enrolled. At the time of the analysis, $200(55.6 \%)$ patients had at least 2 years of follow-up. Table 1 summarizes patients' demographic and baseline clinical characteristics (EDSS score, disease duration, ARR, MRI, MS activity, and JCV status) on the whole cohort and according to groups defined by mean interval between doses (ITT and PP analyses).

The mean interval between doses in the 6 months following the month 24 infusion was 5.2 weeks (median, 4.7 weeks; IQR 4.2-6.0), with a clear bimodal distribution (modes at 4 and 6 weeks; Fig. 1S), and it was associated with individual center strategies (the median was 4.3 weeks in 225 patients from 12 centers and 6.1 in 127 patients from 2 centers). The mean interval between doses was 5.2 weeks (median, 5; IQR 4.3-6) over the whole follow-up.
Two hundred and sixteen patients were in the SID group (median dose interval, first 6 months $=4.3$ weeks [IQR $4.1-$ 4.5]; whole follow-up = 4.4 [IQR 4.1-5]) and 144 in the EID group (median dose interval, first 6 months $=6.2$ weeks [IQR 5.6-6.7]; whole follow-up = 6 [IQR 5.4-6.5]) (Fig. 1).

The differences of prenatalizumab and baseline characteristics of patients in the 2 treatment regimens are reported in Table 1. EID patients had a higher ARR (1.17 vs 0.99; $p=$ 0.003 ) and EDSS at natalizumab start ( 3 vs $2.5 ; p=0.003$ ) but showed a higher decrease during 24 doses $(-0.43 v s-0.14$; $p=0.002$ ). These results do not suggest a higher activity of SID vs EID at baseline.

ARR during follow-up adjusting for all the baseline variables (age, disease duration, relapses in the 2 years prenatalizumab, relapses during natalizumab, baseline EDSS, EDSS change during natalizumab, number of previous treatments) in the ITT analysis was $0.060(95 \% \mathrm{CI}=0.033$ $0.087)$ in the SID group, and it was $0.039(95 \% \mathrm{CI}=0.017-$ 0.063 ) in the EID group. The noninferiority of EID versus SID was satisfied (Fig. 1, left panel; Table 1S). The mean time to first relapse was 1.88 years in the SID group and 1.90 years in the EID group (Fig. 2; $\mathrm{HR}=0.71(95 \% \mathrm{CI}=0.34-1.74, p=$ 0.36 ; after adjusting for age, disease duration, relapses in the 2 years prenatalizumab, relapses during natalizumab, baseline EDSS, EDSS change during natalizumab, number of previous treatments)).

In the PP analysis, patients were classified in the SID or EID group according to the mean intervals between doses received from baseline to the end of follow-up (or the first relapse), whichever came first. One hundred and eighty-two patients were in the SID group (median dose interval = 4.3 weeks) and 178 in the EID group (median dose interval = 6.0 weeks). ARR during follow-up adjusting for all the baseline variables in the PP analysis was $0.067(95 \% \mathrm{CI}=0.038$ $0.096)$ in the SID group, and it was $0.039(95 \% \mathrm{CI}=0.016$ 0.062) in the EID group (Fig. 1, right panel; Table 1S). The mean time to first relapse was 1.87 years in the SID group and 1.92 years in the EID group (Fig. 2; $\mathrm{HR}=0.58(95 \% \mathrm{CI}=$ $0.28-1.18, p=0.13$; after adjusting for age, disease duration, relapses in the 2 years prenatalizumab, relapses during natalizumab, baseline EDSS, EDSS change during natalizumab, number of previous treatments)).

The results were stable at the sensitivity analyses. The ARR was $0.062(95 \% \mathrm{CI}=0.029-0.095)$ in the SID group, and it was $0.021(95 \% \mathrm{CI}=0.003-0.039)$ in the EID group when the 2 groups were defined removing patients with a mean interval between doses in the range 32 to 38 days. When the interval dose was analysed as a continuous variable, the effect of the interval dose length on the ARR was not statistically significant $(\mathrm{RR}=0.93$ (95\% CI $0.64-1.36, p=0.73)$. No PML or other serious adverse events have been reported during the follow-up. 
Table 1 Patients' demographic and baseline clinical characteristics on all samples and according to interval dose groups

\begin{tabular}{|c|c|c|c|c|c|c|c|}
\hline & \multirow{2}{*}{$\begin{array}{l}\text { All patients } \\
(n=360)\end{array}$} & \multicolumn{3}{|c|}{ Intention-to-treat (ITT) } & \multicolumn{3}{|c|}{ Per-protocol (PP) } \\
\hline & & $\begin{array}{l}\text { SID } \\
(n=216)\end{array}$ & $\begin{array}{l}\text { EID } \\
(n=144)\end{array}$ & $p$ value & $\begin{array}{l}\text { SID } \\
(n=182)\end{array}$ & $\begin{array}{l}\text { EID } \\
(n=178)\end{array}$ & $p$ value \\
\hline Age, mean (SD) & $34.3(10.3)$ & $34.3(10.7)$ & $34.3(9.8)$ & 0.73 & $33.8(10.7)$ & $34.9(10)$ & 0.31 \\
\hline Females, $n(\%)$ & $254(70.6)$ & $147(68.1)$ & $107(74.3)$ & 0.24 & $128(70.3)$ & $126(70.8)$ & 0.92 \\
\hline Disease duration (years), median (IQR) & $4.6(1.2-8.8)$ & $3.8(0.9-8.5)$ & $5.4(1.9-9.1)$ & 0.085 & $3.1(0.8-7.5)$ & $5.5(1.8-9.8)$ & 0.002 \\
\hline EDSS prenatalizumab start, median (IQR) & $2.5(2-4)$ & $2.5(1.5-3.5)$ & $3(2-4)$ & 0.003 & $2.5(1.5-4)$ & $3(2-4)$ & 0.014 \\
\hline EDSS change during natalizumab, mean (SD) & $-0.26(0.89)$ & $-0.14(0.89)$ & $-0.43(0.86)$ & 0.002 & $-0.09(0.85)$ & $-0.43(0.89)$ & 0.001 \\
\hline ARR during natalizumab, mean (SD) & $0.069(0.32)$ & $0.065(0.37)$ & $0.083(0.42)$ & 0.46 & $0.058(0.37)$ & $0.09(0.41)$ & 0.12 \\
\hline ARR 2 year pre natalizumab, mean (SD) & $1.06(0.64)$ & $0.99(0.61)$ & $1.17(0.67)$ & 0.003 & $0.93(0.58)$ & $1.20(0.67)$ & $<0.001$ \\
\hline $\mathrm{JCV}+$ at month $24, n(\%)$ & $145 / 354(41)$ & $\begin{array}{r}71 / 210 \\
(33.8)\end{array}$ & $\begin{array}{r}74 / 144 \\
(51.4)\end{array}$ & 0.001 & $\begin{array}{l}53 / 177 \\
(29.9)\end{array}$ & $92 / 177(52)$ & $<0.001$ \\
\hline $\begin{array}{l}\text { Number of previous treatments, mean; median } \\
\text { (range) }\end{array}$ & $1(0-7)$ & $1.23 ; 1(0-5)$ & $1.68 ; 1(0-7)$ & 0.001 & $1.18 ; 1(0-5)$ & $1.65 ; 1(0-7)$ & 0.001 \\
\hline Active lesions at month $24, n / N(\%)$ & $60 / 348(17.2)$ & $\begin{array}{l}53 / 206 \\
(25.7)\end{array}$ & $7 / 142(4.9)$ & $<0.001$ & $\begin{array}{l}56 / 176 \\
(31.8)\end{array}$ & $4 / 172(2.3)$ & $<0.001$ \\
\hline
\end{tabular}

$\mathrm{SD}=$ standard deviation, $\mathrm{IQR}=$ interquartile range, EDSS $=$ Expanded Disability Status Scale, $\mathrm{ARR}=$ annualized relapse rate, $\mathrm{JCV}=\mathrm{JC}$ virus, $\mathrm{ITT}=$ intention-to-treat, $\mathrm{PP}=$ per-protocol, $\mathrm{SID}=$ standard interval dose, EID = extended interval dose

Using the PS method to adjust for differences between SID and EID on baseline variables, similar results were obtained: in the ITT analysis, the ARR was $0.072(95 \% \mathrm{CI}=0.042-0.103)$ in the SID and 0.045 (95\% CI 0.015-0.076) in the EID whereas in the PP analysis, the ARR was, respectively, $0.076(95 \% \mathrm{CI}=$ $0.042-0.109)$ and $0.040(95 \% \mathrm{CI}=0.016-0.065)$.

\section{Discussion}

Our spontaneous, retrospective, observational multicenter study allowed us to evaluate the efficacy of an extended regimen dose after the 24th natalizumab dose in patients with R-MS with a PML risk due to JCV seropositivity and number of natalizumab administrations received.

Natalizumab is a highly effective drug for R-MS for the outcome of ARR [24]. The use of natalizumab is however burdened by the risk of developing PML, particularly after the 24th administration [25]. As of December 2017, a total of 753 confirmed cases of natalizumab-associated PML cases had been reported [26]. Considering the significant PML risk, as of November 30,2017, the overall PML incidence is 4.19 per 1000 patients (95\% CI 3.89-4.49 per 1000 patients) [26], and according to EMA recommendations [27], neurologists
Fig. 1 Annualized relapse rate over 2 years according to interval dose. ITT = intention-to-treat, PP $=$ per-protocol, $\mathrm{SID}=$ standard interval dose, EID = extended interval dose
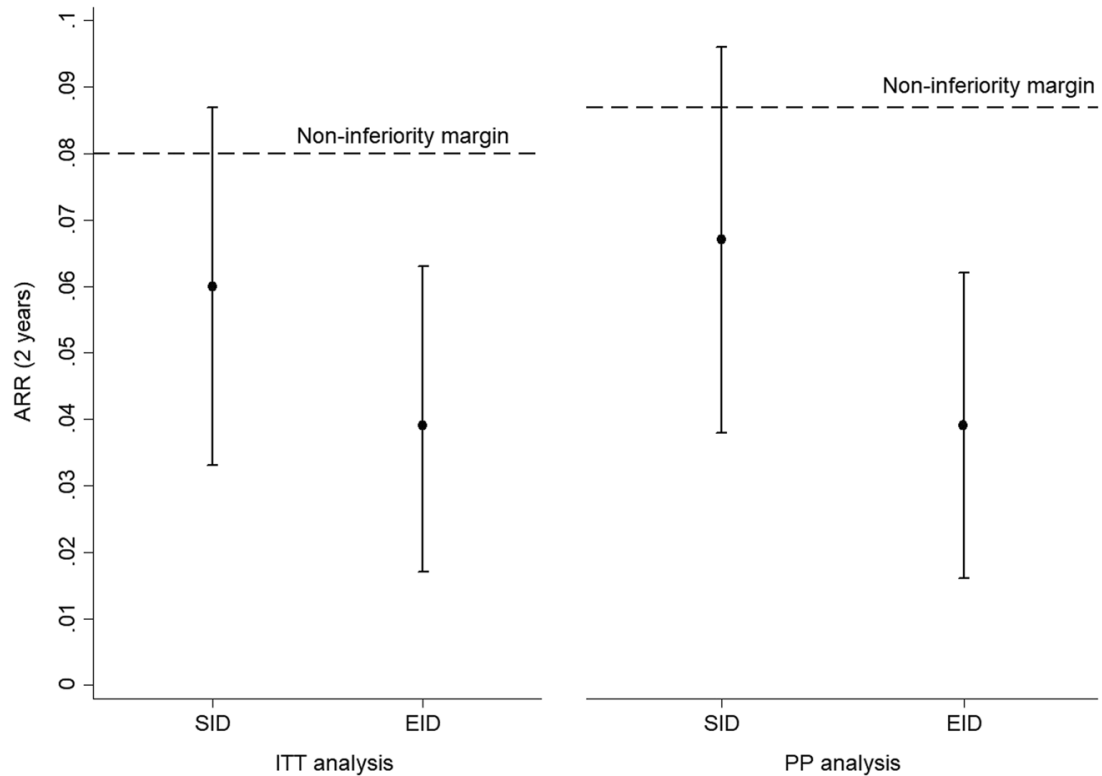

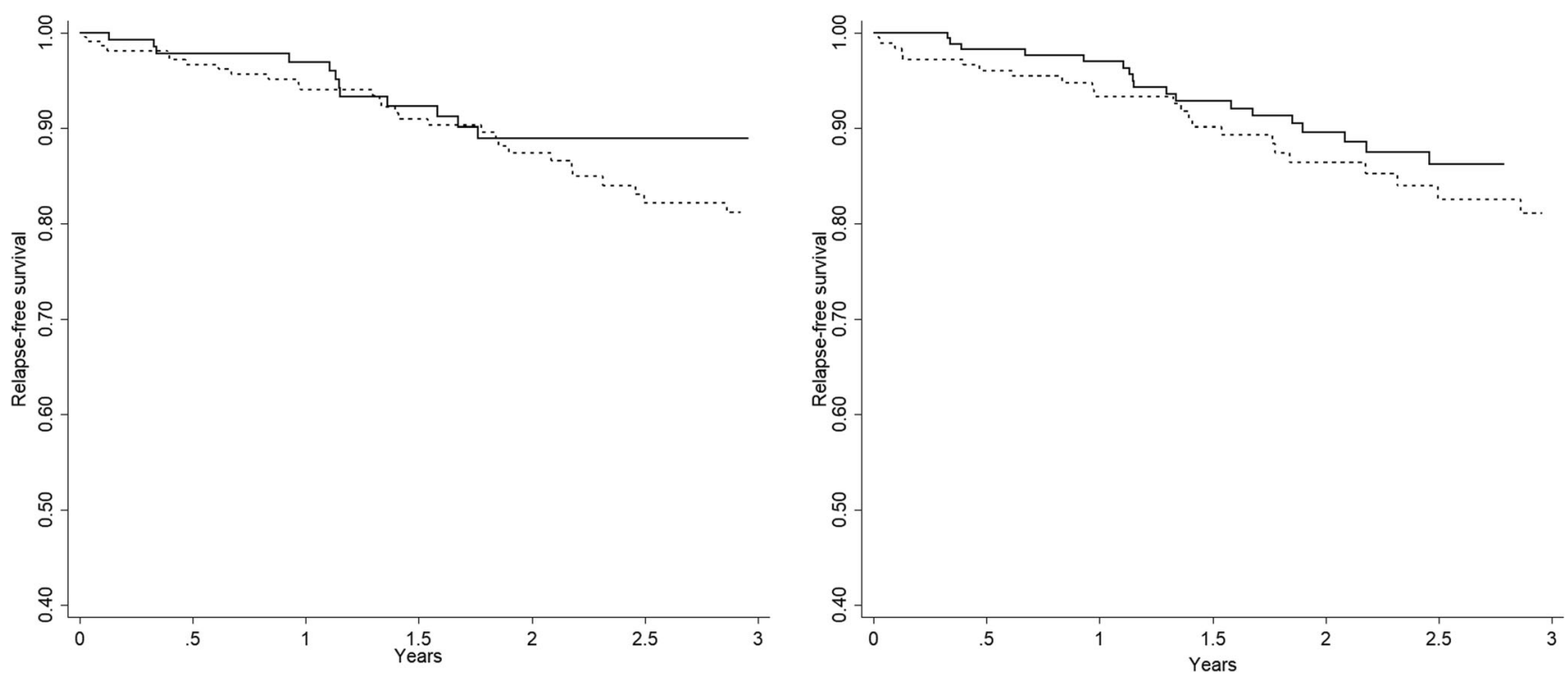

Fig. 2 Time to first relapse according to interval dose. Solid line: standard interval dose; dash line: extended interval dose

have to re-evaluate natalizumab treatment after 24 doses. Many studies and single case reports showed that after natalizumab, withdrawal disease activity could worse than prenatalizumab status [28-31] and also, switching to firstline treatments could increase the MS relapse rate [32]. Based on these premises, many studies have been carried out to detect the best strategy to minimize both PML and rebound risk [32]. Some clinicians throughout the world started to prescribe various EID schedules to treat highly active R-MS patients who decided to continue natalizumab after the 24th administration, in order to low PML risk, although a higher concentration of natalizumab is not definitively and clearly associated with a higher PML rate [15, 33], although a recent study demonstrates that an EID schedule is associated with a clinically and statistically significant lower risk of PML than SID in JCV antibody-positive patients [34].

The purpose of this study was to assess the noninferiority of a natalizumab EID regimen versus the 4-week SID schedule. The results show that there is no evidence of a reduced efficacy of natalizumab in an EID setting, from a median of 4.3 to a median of 6.2 weeks, with comparable results seen across all measured outcomes, both in the ITT and in the PP analyses.

Some significant difference between SID and EID patients on ARR and EDSS before treatment start period and during natalizumab administration was observed with a higher disease activity of the EID group. For this reason, the lack of differences between EID and SID on disease activity during follow-up should not be influenced by baseline characteristics.

A limitation of our study is the lack of a parallel pharmacokinetics analysis of natalizumab in both groups, in order to asses both concentrations of the drug and $\alpha 4 \beta 1$ integrin receptor saturation along time.
Also, we lack a systematic MRI assessment to check whether efficacy of EID is preserved also on radiological activity; this is a consequence of the retrospective nature of the study and of the different timings with which the brain MRI are performed in each center.

Neither severe adverse events nor PML have been described, but a further prolonged analysis is necessary to assess also a possible PML reduction risk in an EID larger cohort of patients. A recent study on a large cohort of natalizumabtreated patients showed a $94 \%$ reduction of PML risk in an EID setting versus a SID one in the primary analysis and an $88 \%$ reduction in risk in the secondary analysis (both $p<0.0001$ ) [34].

According to previous studies that showed that natalizumab discontinuation could be hazardous [30, 32, 35], the EID schedule appears as a reasonable treatment, able to maintain the high efficacy of natalizumab. Keeping the same efficacy, reducing the frequency of natalizumab administration could, in addition, improve the quality of life of MS patients, making them less tied to the hospital administration of the drug. At the same time, an EID schedule could lead to a reduction of hospital costs for the purchase and administration of therapy would be observed.

\section{Conclusion}

Our study shows that clinical MS reactivation does not occur more frequently in patients in the EID group. These results come from a large, retrospective multicenter study that can have a very useful implication in clinical practice. According to our results, continuing natalizumab in an EID setting seems an efficacious therapeutic strategy in patients with highly active MS who decide to continue natalizumab 24 doses. The 
absence of evidence of a reduced efficacy of the EID strategy could be taken into account in planning further studies aimed at impacting the decision process about the therapeutic management of JCV-positive patients after 24 doses of natalizumab.

In conclusion, this observation confirms previous results $[15,21,33]$ and together with the emerging evidence of a reduced risk of PML associated to an EID, supports the need of a randomized study to change the standard of the natalizumab dosing schedule. In November 2018, a randomized, controlled open-label phase $3 \mathrm{~b}$ study started to enrol MS patients with the primary objective to evaluate the efficacy of a natalizumab EID in subjects who have previously been treated with a natalizumab SID for at least 12 months, in relation to continued SID treatment [36].

\section{Compliance with Ethical Standards}

Ethical committees of each participating hospital or university approved the study protocol.

Required Author Forms Disclosure forms provided by the authors are available with the online version of this article.

\section{References}

1. Stüve O, Bennett JL. Pharmacological properties, toxicology and scientific rationale for the use of natalizumab (Tysabriß) in inflammatory diseases. CNS Drug Rev. 2007 Spring;13(1):79-95.

2. Polman CH, O’Connor PW, Havrdova E, Hutchinson M, Kappos $\mathrm{L}$, Miller DH, et al. A randomized, placebo-controlled trial of natalizumab for relapsing multiple sclerosis. N Engl J Med. 2006;354(9):899-910. Available from: http://www.ncbi.nlm.nih. gov/pubmed/16510744. Accessed 27 May 2019

3. Rudick RA, Stuart WH, Calabresi PA, Confavreux C, Galetta SL, Radue E-W, et al. Natalizumab plus Interferon Beta-1a for Relapsing Multiple Sclerosis. N Engl J Med. 2006;354(9):911-23.

4. Sehr T, Proschmann U, Thomas K, Marggraf M, Straube E, Reichmann $\mathrm{H}$, et al. New insights into the pharmacokinetics and pharmacodynamics of natalizumab treatment for patients with multiple sclerosis, obtained from clinical and in vitro studies. $\mathrm{J}$ Neuroinflammation. 2016;13(1):164. https://doi.org/10.1186/ s12974-016-0635-2

5. Kleinschmidt-DeMasters BK, Tyler KL. Progressive Multifocal Leukoencephalopathy Complicating Treatment with Natalizumab and Interferon Beta-1a for Multiple Sclerosis. N Engl J Med 2005;353(4):369-74.

6. Langer-Gould A, Atlas SW, Green AJ, Bollen AW, Pelletier D. Progressive multifocal leukoencephalopathy in a patient treated with natalizumab. N Engl J Med 2005;353(4):375-81.

7. Tan CS, Koralnik IJ. Progressive multifocal leukoencephalopathy and other disorders caused by JC virus: clinical features and pathogenesis. Lancet Neurol. 2010;9:425-37.

8. Rudick RA, Sandrock A. Natalizumab: $\alpha 4$-integrin antagonist selective adhesion molecule inhibitors for MS. Vol. 4, Expert Review of Neurotherapeutics. 2004. p. 571-80.

9. Khatri BO, Man S, Giovannoni G, Koo AP, Lee JC, Tucky B, et al. Effect of plasma exchange in accelerating natalizumab clearance and restoring leukocyte function. Neurology. 2009;72(5):402-9.
10. Cofield SS, Salter A, Tyry T, Crowe C, Cutter GR, Fox RJ, et al. Perspectives on marijuana use and effectiveness: A survey of NARCOMS participants. Neurol Clin Pract. 2017;7(4):333-343. https://doi.org/10.1212/CPJ.0000000000000383

11. Rispens T, Vennegoor A, Wolbink GJ, Polman CH, Killestein J. Natalizumab remains detectable in patients with multiple sclerosis long after treatment is stopped. Mult Scler. 2012;18(16):899-901. https://doi.org/10.1177/1352458511431073

12. Wipfler P, Harrer A, Pilz G, Oppermann K, Afazel S, HaschkeBecher E, et al. Natalizumab saturation: Biomarker for individual treatment holiday after natalizumab withdrawal? Acta Neurol Scand. 2014;129(3):e12-5. https://doi.org/10.1111/ane.12182

13. van Kempen ZLE, Leurs CE, Witte BI, de Vries A, Wattjes MP, Rispens T, et al. The majority of natalizumab-treated MS patients have high natalizumab concentrations at time of re-dosing. Mult Scler. 2018;24(6):805-810. https://doi.org/10.1177/ 1352458517708464

14. Tan IL, McArthur JC, Clifford DB, Major EO, Nath A. Immune reconstitution inflammatory syndrome in natalizumab-associated PML. Neurology. 2011;77(11):1061-7.

15. Zhovtis Ryerson L, Frohman TC, Foley J, Kister I, WeinstockGuttman B, Tornatore C, et al. Extended interval dosing of natalizumab in multiple sclerosis. J Neurol Neurosurg Psychiatry 2016;1-5. Available from: http://www.ncbi.nlm.nih.gov/pubmed/ 26917698. Accessed 27 May 2019

16. Fox RJ, Cree BAC, De Sèze J, Gold R, Hartung HP, Jeffery D, et al. MS disease activity in RESTORE: A randomized 24-week natalizumab treatment interruption study. Neurology. 2014;82(17): 1491-8.

17. Harrer A, Pilz G, Einhaeupl M, Oppermann K, Hitzl W, Wipfler P, et al. Lymphocyte subsets show different response patterns to in vivo bound natalizumab-a flow cytometric study on patients with multiple sclerosis. PLoS One. 2012;7(2):e31784. https://doi.org/10. 1371/journal.pone.0031784

18. European Medicines Agency. European Medicines Agency recommends additional measures to better manage risk of progressive multifocal leukoencephalopathy (PML) with Tysabri [Internet]. [cited 2018 Jun 16]. Available from: http:/www.ema.europa.eu/ ema/index.jsp?curl=pages/news and events/news/2010/01/news detail_000987.jsp\&mid=WC0b01 ac058004d5c1

19. Kappos L, Bates D, Edan G, Eraksoy M, Garcia-Merino A, Grigoriadis N, et al. Natalizumab treatment for multiple sclerosis: Updated recommendations for patient selection and monitoring. Lancet Neurol 2011;10(8):745-58. https://doi.org/10.1016/S14744422(11)70149-1

20. Bloomgren G, Richman S, Hotermans C, Subramanyam M, Goelz S, Natarajan A, et al. Risk of Natalizumab-Associated Progressive Multifocal Leukoencephalopathy. N Engl J Med. 2012;366(20): 1870-80. Available from: http://www.nejm.org/doi/abs/10.1056/ NEJMoa1107829. Accessed 27 May 2019

21. Bomprezzi R, Pawate S. Extended interval dosing of natalizumab: A two-center, 7-year experience. Ther Adv Neurol Disord. 2014;7(5):227-31. https://doi.org/10.1177/1756285614540224

22. Thompson AJ, Banwell BL, Barkhof F, Carroll WM, Coetzee T, Comi G, et al. Diagnosis of multiple sclerosis: 2017 revisions of the McDonald criteria. Lancet Neurol 2018;17(2):162-73.

23. Kurtzke JF. Rating neurologic impairment in multiple sclerosis: an expanded disability status scale (EDSS). Neurology. 1983;33(11): 1444-52.

24. Lucchetta RC, Tonin FS, Borba HHL, Leonart LP, Ferreira VL, Bonetti AF, et al. Disease-Modifying Therapies for RelapsingRemitting Multiple Sclerosis: A Network Meta-Analysis. CNS Drugs. 2018. Available from: http://www.ncbi.nlm.nih.gov/ pubmed/30014314. Accessed 27 May 2019

25. Sørensen PS, Bertolotto A, Edan G, Giovannoni G, Gold R, Havrdova E, et al. Risk stratification for progressive multifocal 
leukoencephalopathy in patients treated with natalizumab. Mult Scler 2012;18(2):143-52. Available from: http://www.ncbi.nlm. nih.gov/pubmed/22312009. Accessed 27 May 2019

26. https://chefarztfrau.de/?page_id=716 [Internet]. [cited 2018 May 31]. Available from: https://chefarztfrau.de/?page id $=716$

27. EMA confirms recommendations to minimise risk of brain infection PML with Tysabri - EMA/137488/2016.

28. O'Connor PW, Goodman A, Kappos L, Lublin FD, Miller DH, Polman C, et al. Disease activity return during natalizumab treatment interruption in patients with multiple sclerosis. Neurology. 2011;76(22):1858-65.

29. Berger JR, Centonze D, Comi G, Confavreux C, Cutter G, Giovannoni $\mathrm{G}$, et al. Considerations on discontinuing natalizumab for the treatment of multiple sclerosis. Ann Neurol 2010;68(3): 409-11. Available from: http://www.ncbi.nlm.nih.gov/pubmed/ 20818795

30. Papeix C, Vukusic S, Casey R, Debard N, Stankoff B, Mrejen S, et al. Risk of relapse after natalizumab withdrawal: Results from the French TYSEDMUS cohort. Neurol Neuroimmunol Neuroinflammation 2016;3(6):e297. Available from: http://www. ncbi.nlm.nih.gov/pubmed/27844037

31. González-Suarez I, Rodríguez de Antonio L, Orviz A, MorenoGarcía S, Valle-Arcos MD, Matias-Guiu JA, et al. Catastrophic outcome of patients with a rebound after Natalizumab treatment discontinuation. Brain Behav 2017;7(4):1-6.

32. Clerico M, Schiavetti I, De Mercanti SF, Piazza F, Gned D, Brescia Morra V, et al. Treatment of Relapsing-Remitting Multiple Sclerosis After 24 Doses of Natalizumab: Evidence From an Italian Spontaneous, Prospective, and Observational Study (the
TY-STOP Study). JAMA Neurol 2014;71(8):954-60. Available from: http://www.ncbi.nlm.nih.gov/pubmed/24977406

33. Van Kempen ZLE, Leurs CE, Vennegoor A, Wattjes MP, Rispens T, Uitdehaag BMJ, et al. Natalizumab-associated progressive multifocal leukoencephalopathy is not preceded by elevated drug concentrations. Mult Scler J. 2017;23(7):995-999. https://doi.org/10.1177/ 1352458516684023

34. Zhovtis, Ryerson L, Foley J CIKICGMRGJLXREYBRZHCHPRCN. Natalizumab Extended Interval Dosing (EID) Is Associated with a Significant Reduction in Progressive Multifocal Leukoencephalopathy (PML) Risk Compared with Standard Interval Dosing (SID): Analyses of TOUCH® Prescribing Program Data. In: American Academy of Neurology. 2018.

35. Sorensen PS, Koch-Henriksen N, Petersen T, Ravnborg M, Oturai A, Sellebjerg F. Recurrence or rebound of clinical relapses after discontinuation of natalizumab therapy in highly active MS patients. J Neurol . 2014;261(6):1170-7. Available from: http:// www.ncbi.nlm.nih.gov/pubmed/24728334

36. A Study to Evaluate Efficacy, Safety, and Tolerability of 6-Week Extended Interval Dosing of Natalizumab (BG00002) in Participants With Relapsing-Remitting Multiple Sclerosis (RRMS) Switching From Treatment With 4-Week Natalizumab Standard Interval Dosing [Internet]. [cited 2019 Jul 30]. Available from: https://clinicaltrials.gov/ct2/show/NCT03689972?term= NCT03689972\&rank=1

Publisher's Note Springer Nature remains neutral with regard to jurisdictional claims in published maps and institutional affiliations.

\section{Affiliations}

\section{Marinella Clerico ${ }^{1}$-Stefania Federica De Mercanti ${ }^{1}$ - Alessio Signori ${ }^{2} \cdot$ Marco ludicello $^{1} \cdot$ Cinzia Cordioli $^{3}$. Elisabetta Signoriello ${ }^{4}$. Giacomo Lus ${ }^{4}$. Simona Bonavita ${ }^{5}$. Luigi Lavorgna ${ }^{5}$. Giorgia Teresa Maniscalco ${ }^{6}$. Erica Curti ${ }^{7}$ - Lorena Lorefice ${ }^{8}$. Eleonora Cocco ${ }^{8} \cdot$ Viviana Nociti $^{9} \cdot$ Massimiliano Mirabella ${ }^{9} \cdot$ Damiano Baroncini ${ }^{10}$. Giorgia Mataluni ${ }^{11}$. Doriana Landi ${ }^{11}$. Martina Petruzzo ${ }^{12}$. Roberta Lanzillo ${ }^{12} \cdot$ Ilaria Gandoglia ${ }^{13}$. Alice Laroni $^{14}$. Rita Frangiamore ${ }^{15}$. Arianna Sartori $^{16}$. Paola Cavalla ${ }^{17}$. Gianfranco Costantini ${ }^{17} \cdot$ Maria Pia Sormani $^{2}$. Ruggero Capra ${ }^{3}$}

1 Clinical and Biological Sciences Department, Neurology Unit, University of Torino, San Luigi Gonzaga Hospital, Regione Gonzole, 10, Orbassano, 10043 Turin, Italy

2 Department of Health Sciences, Section of Biostatistics, University of Genova, Genoa, Italy

3 Multiple Sclerosis Center, Spedali Civili of Brescia, Presidio di Montichiari, Brescia, Italy

4 Department of Medical, Surgical, Neurological, Metabolic and Aging Sciences, Second University of Naples, Naples, Italy

5 Clinic of Neurology, AOU - University of Campania "Luigi Vanvitelli”, Naples, Italy

6 Neurological Clinic and Multiple Sclerosis Centre of "AORN A. Cardarelli”, Naples, Italy

7 Neurology Unit, Department of Medicine and Surgery, University of Parma, Parma, Italy
8 Multiple Sclerosis Center, Binaghi Hospital, Department of Medical Sciences and Public Health, University of Cagliari, Cagliari, Italy

9 Multiple Sclerosis Center, Neuroscience Area, Neuroscience, Aging, Head and Neck and Orthopaedics Sciences Department, Fondazione Policlinico Universitario Gemelli, Rome, Italy

10 Centro Sclerosi Multipla - Presidio ospedaliero di Gallarate - ASST Valle Olona, Gallarate, Italy

11 UOSD Centro di Riferimento Regionale Sclerosi Multipla Dipartimento di Neuroscienze Policlinico Tor Vergata, Rome, Italy

12 Department of Neurosciences, Reproductive Sciences and Odontostomatology, Multiple Sclerosis Centre, Federico II University, Naples, Italy

13 Department of Neuroscience, Rehabilitation, Ophthalmology, Genetics, Maternal and Child Health and Center of Excellence for Biomedical Research, University of Genova, Genoa, Italy 
14 IRCCS Ospedale Policlinico San Martino, Genoa, Italy

15 Department of Neuroimmunology and Neuromuscular Diseases, Neurological Institute C. Besta, IRCCS Foundation, Milan, Italy
16 Neurology Unit, Azienda Sanitaria Univeristaria Integrata Clinica Neurologica, Azienda Ospedaliero-Universitaria Ospedali Riuniti di Trieste, Trieste, Italy

17 Department of Neuroscience, Città della Salute e della Scienza di Torino University Hospital, Turin, Italy 